\title{
Nucleotide Polymorphisms in the Canine Noggin Gene and Their Distribution Among Dog (Canis lupus familiaris) Breeds
}

\author{
Yuji Ishii • Tatsuya Takizawa • Hiroshi Iwasaki • \\ Yukihiro Fujita · Masaru Murakami • \\ Jay C. Groppe $\cdot$ Kazuaki Tanaka
}

Received: 17 March 2010/Accepted: 28 April 2011/Published online: 1 September 2011

(C) The Author(s) 2011. This article is published with open access at Springerlink.com

\begin{abstract}
Noggin (NOG) is an important regulator for the signaling of bone morphogenetic proteins. In this study, we sequenced the complete coding sequence of the canine $N O G$ gene and characterized the nucleotide polymorphisms. The sequence length varied from 717 to $729 \mathrm{bp}$, depending on the number of a 6-bp tandem repeat unit (GGCGCG), an insertion that has not been observed in other mammalian $N O G$ genes investigated to date. It results in extensions of (Gly-Ala) $3-5$ in the putative NOG protein. To survey the distribution of these tandem repeat polymorphisms, we analyzed 126 individuals in seven dog breeds. We identified only three alleles: $(\mathrm{GGCGCG})_{3}$, (GGCGCG) $)_{4}$, and (GGCGCG) $)_{5}$. Although the allele frequencies were remarkably different among the breeds, the three alleles were present in all seven of the breeds and did not show any deviation from HardyWeinberg equilibrium.
\end{abstract}

Electronic supplementary material The online version of this article (doi:

$10.1007 / \mathrm{s} 10528-011-9453-5)$ contains supplementary material, which is available to authorized users.

Y. Ishii · T. Takizawa $\cdot$ H. Iwasaki $\cdot$ K. Tanaka $(\bowtie)$

Laboratory of Animal Biotechnology, School of Veterinary Medicine, Azabu University, Chuou-ku, Sagamihara 252-5201, Japan

e-mail: tanakak@azabu-u.ac.jp

Y. Fujita

Laboratory of Veterinary Surgery 2, School of Veterinary Medicine, Azabu University, Chuou-ku, Sagamihara 252-5201, Japan

\section{Murakami}

Laboratory of Molecular Biology, School of Veterinary Medicine, Azabu University, Chuou-ku, Sagamihara 252-5201, Japan

J. C. Groppe

Department of Biomedical Sciences, Baylor College of Dentistry, TAMHSC, Dallas, TX 75246, USA 
Keywords Noggin · Dog · Canis lupus familiaris · Polymorphisms · VNTR

\section{Introduction}

Noggin (NOG) is a member of the bone morphogenetic protein (BMP) family and a major extracellular antagonist of the BMP. NOG regulates dorsal induction and joint formation in embryonic development by blocking BMP signaling (Smith and Harland 1992; Zimmerman et al. 1996; Brunet et al. 1998; McMahon et al. 1998; Canalis et al. 2003). NOG also controls bone regeneration and homeostasis in adults (Canalis et al. 2003; Canalis 2009). The coding sequences of mammalian NOG genes are contained in a single exon and are highly conserved among species (Valenzuela et al. 1995). Nucleotide and amino acid sequence similarities are more than $90 \%$ among humans, mice, and other mammals (Valenzuela et al. 1995). In humans, several point mutations in the $N O G$ gene have been linked to congenital skeletal malformations, for example, proximal symphalangism or multiple synostoses syndrome (Gong et al. 1999; Marcelino et al. 2001; Hirshoren et al. 2008). Therefore, the $N O G$ gene is a potential candidate for involvement in congenital skeletal malformations in domesticated dogs. Partial DNA sequences at this locus have been defined by the Canine Genome Project (NW_876332; Canis familiaris chromosome 9 genomic contig). To the best of our knowledge, no nucleotide polymorphisms have been described in the canine $N O G$ gene. In the present study, we first determined the DNA sequence of the complete coding sequence of the canine $N O G$ gene, to scan for all the possible nucleotide polymorphisms encoded at this locus. We subsequently surveyed the allelic distribution of the variable number of tandem repeats (VNTRs) that were identified in the canine NOG gene.

\section{Materials and Methods}

Tissue and nail samples from non-sibling dogs were collected at veterinary hospitals and dog-grooming shops in Japan; 127 samples were obtained from 24 long-haired Miniature Dachshunds, 25 long-haired Chihuahuas, 23 Miniature Poodles, 19 Shih Tzus, 13 Papillons, 12 Malteses, 10 Yorkshire Terriers, and 1 Beagle. Genomic DNA was extracted from tissue or nails by a standard phenol-chloroform extraction method (Sambrook et al. 1989) or using the QuickGene DNA tissue kit (Fuji Film, Tokyo, Japan).

\section{Amplification and Sequencing of Canine NOG Coding Sequence}

Four animals from three breeds were selected for DNA sequence determination of the complete coding sequence for the canine $N O G$ gene (one Beagle, two Miniature Dachshunds, and one Chihuahua). Primers for polymerase chain reaction (PCR) and sequencing were designed from dog genome draft sequences (chromosome 9, NW_876332); rat (NM_012990), mouse (NM_008711), and human (NM_005450) $N O G$ gene sequences (Table 1). The primer sets of CanNOG-F1 and CanNOG-R1 
Table 1 Primers for canine $N O G$ gene

\begin{tabular}{lll}
\hline Primer & Sequence $\left(5^{\prime}\right.$ to $\left.3^{\prime}\right)$ & Position on NW_876332 \\
\hline CanNOG-F1 & TGGTGATGGAGCTGAAAGTG & $13273923-13273942$ \\
CanNOG-R1 & ACCACAGCCACATCTGTAACTTC & $13274986-13275008$ \\
CanNOG-F2 & ACCCGGACCCTATCTTTGAC & $13274685-13274704$ \\
CanNOG-R2 & TTTCTGGCTACAGAGACCTAGCT & $13275418-13275440$ \\
CanNOG-S1 & GAAGTTACAGATGTGGCTGTGGT & Reverse complement of R1 \\
CanNOG-S2 & GTCAAAGATAGGGTCCGGGT & Reverse complement of F2 \\
\hline
\end{tabular}

a Canis familiaris chromosome 9 genomic contig, whole genome shotgun sequence

b Primers used only for sequencing

produce about $1.1 \mathrm{kbp}$ DNA products, whereas the primer sets of CanNOG-F2 and CanNOG-R2 produce about $700 \mathrm{bp}$. The two products overlapped with one another in the canine $N O G$ coding sequence.

The PCR was performed according to the manufacturer's instructions for PrimeStar HS DNA polymerase with GC buffer (Takara Biotechnology, Shiga, Japan). The program included first denaturation at $98^{\circ} \mathrm{C}$ for $2 \mathrm{~min} ; 35$ cycles of denaturation at $98^{\circ} \mathrm{C}$ for $20 \mathrm{~s}$, annealing at $55^{\circ} \mathrm{C}$ for $5 \mathrm{~s}$, and extension at $72^{\circ} \mathrm{C}$ for $60 \mathrm{~s}$; and final extension at $72^{\circ} \mathrm{C}$ for $3 \mathrm{~min}$. The amplified products of each reaction were cloned into the plasmid pCR-Blunt II-TOPO using the Zero Blunt TOPO PCR Cloning kit (Invitrogen, Carlsbad, USA). At least five clones were isolated from each individual and sequenced using the Big Dye Terminator version 3.1 Cycle Sequencing Kit and ABI 3100 sequencer (Applied Biosystems, Foster City, USA) with M13 universal primers and additional sequencing primers (Table 1).

Detection of the VNTR Polymorphisms in the NOG Coding Sequence

In order to survey the distribution of the VNTR alleles in dog breeds, we analyzed 126 animals from seven breeds (Table 2). The $300 \mathrm{bp} \mathrm{DNA}$ products of the canine NOG gene, including the 6-bp tandem repeats (GGCGCG), were PCR-amplified

Table 2 Genotype and allele frequency of (GGCGCG) VNTR in canine $N O G$ gene among seven dog breeds

\begin{tabular}{|c|c|c|c|c|c|c|c|c|c|}
\hline \multirow[t]{2}{*}{ Breed (number of animals sampled) } & \multicolumn{6}{|c|}{ Genotype } & \multicolumn{3}{|c|}{ Allele frequency } \\
\hline & 3 & 4 & 5 & $3 / 4$ & $3 / 5$ & $4 / 5$ & 3 & 4 & 5 \\
\hline Miniature Dachshund, long hair (24) & 1 & 7 & 0 & 7 & 7 & 2 & 0.333 & 0.479 & 0.188 \\
\hline Chihuahua, long hair (25) & 3 & 0 & 6 & 5 & 7 & 4 & 0.360 & 0.180 & 0.460 \\
\hline Miniature poodle (23) & 2 & 2 & 2 & 5 & 6 & 6 & 0.326 & 0.326 & 0.348 \\
\hline Shih Tzu (19) & 9 & 0 & 2 & 3 & 4 & 1 & 0.658 & 0.105 & 0.237 \\
\hline Papillon (13) & 5 & 0 & 0 & 2 & 4 & 2 & 0.615 & 0.154 & 0.231 \\
\hline Maltese (12) & 2 & 1 & 0 & 0 & 6 & 3 & 0.417 & 0.208 & 0.375 \\
\hline Yorkshire Terrier (10) & 8 & 0 & 0 & 1 & 1 & 0 & 0.900 & 0.050 & 0.050 \\
\hline
\end{tabular}


using primer sets of CanNOG-F2 and CanNOG-R1 and then purified with the High Pure PCR Product Purification Kit (Roche Applied Science, Mannheim, Germany). Purified DNAs were sequenced directly with the primer CanNOG-F2 as described above. Heterozygous individuals that had a different number of tandem repeats were genotyped from their electropherograms.

\section{Results and Discussion}

In four domestic dogs, we sequenced the entire coding region of the NOG gene, which included an unidentified region of 91 nucleotides in NW_876332. These nucleotide sequences were entered into the DDBJ, EMBL, and GenBank nucleotide databases with accession numbers AB544074-AB544077. After multiple alignment of canine sequences with human NOG (NM_005450) and mouse Nog (NM_008711), we found that the coding sequence of the canine $N O G$ gene is longer than that humans and mice. The coding sequence of the human and mouse genes is consistently $699 \mathrm{bp}$ in length. The coding sequence in the four dogs showed three polymorphisms, of 717, 723, and $729 \mathrm{bp}$, caused by inserted sequences of 18, 24, and $30 \mathrm{bp}$, respectively. These inserted sequences in the canine NOG gene were constructed by different numbers of the 6-bp tandem repeats (GGCGCG) unit (Fig. 1). These GGCGCG tandem repeats immediately preceded the inserted sequences. Thus, these sequence-length polymorphisms are the result of the duplicated insertion of this repeat unit. The insertions of tandem repeats were aligned with the reading frame of the $N O G$ gene and found to encode a Gly-Ala repeat

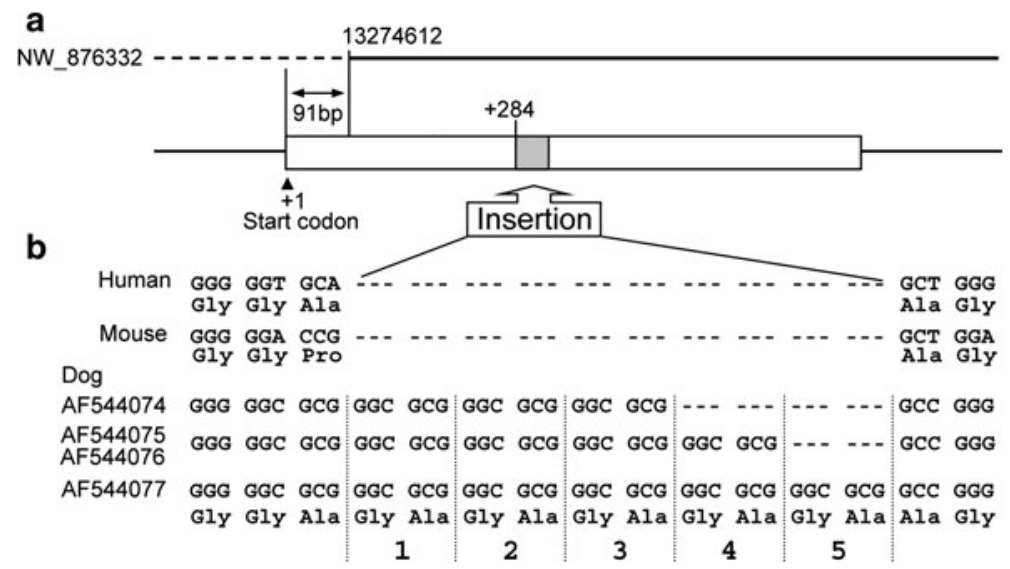

Fig. 1 The coding region of the canine $N O G$ gene and polymorphisms detected in this study. a Structure of the coding region of the canine $N O G$ gene. The solid upper line indicates the partial sequence of canine chromosome 9 (NW_876332), and the broken line represents the undeciphered region in NW_876332. The start codon of canine NOG was located in the unidentified region, -91 bp from position 13274612 on NW_876332. The white blocks of the diagram represent the canine NOG coding sequence, and the shaded block indicates the region of the inserted sequence. b The nucleotide and amino acid sequences around the canine-specific insertion. The repeating units are ruled off by vertical lines. The numbers at the bottom indicate the number of repetitions. Repetition of GGCGCG was translated into the amino acids Gly-Ala 


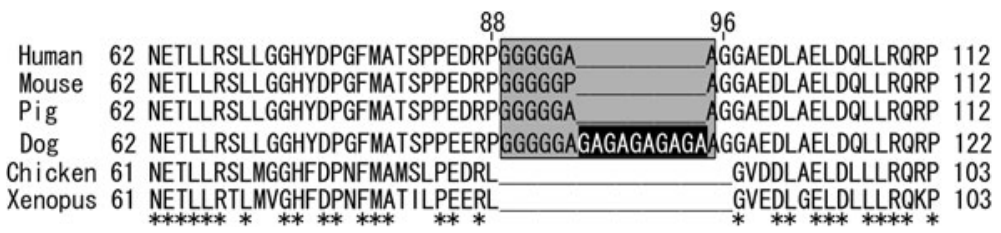

Fig. 2 Comparison of partial amino acid sequences of vertebrate Noggin. Asterisks indicate the conserved amino acids among the six species. The gray box encloses the polyglycine loop (Gly-GlyGly-Gly-Gly-Ala-Ala) insertion, which is present only in mammalian Noggin (Groppe et al. 2002). The highlighted letters indicate extension of the Gly-Ala repeat in the canine Noggin. The Gly-Ala repeat is connected to the polyglycine loop. Citation of sequences: human NM_005450, mouse NM_008711, pig NM_001143691, dog AF544077, chicken NM_204123, and Xenopus NM_00185644

(Fig. 1). These $(\text { Gly-Ala) })_{3-5}$ insertions are located in the polyglycine loop which is present only in mammalian Noggin (Fig. 2). The polyglycine loop is the most flexible part of the mammalian Noggin protein, as a complex with the BMP ligand (Groppe et al. 2002). Excluding these inserted-sequence regions, the coding sequence of the canine $N O G$ gene displayed $96.7 \%$ sequence similarity with the nucleotides of human NOG and $94.0 \%$ with mouse Nog. No other polymorphic sites were found among the four canine $N O G$ sequences, except the above-mentioned inserted sequences.

We sequenced part of the $N O G$ gene, including the tandem repeat (GGCGCG) , in 126 dogs from seven breeds, and identified three alleles, (GGCGCG) , $_{3}$, $(\mathrm{GGCGCG})_{4}$, and (GGCGCG) $)_{5}$, among these individuals (Table 2). The genotypic and allelic frequencies of these VNTR alleles did not deviate from Hardy-Weinberg equilibrium among the seven breeds. Although the three alleles of the VNTR were present in all the dog breeds analyzed in this study, distributions varied widely among the breeds. (GGCGCG) $)_{4}$ had the highest frequency in the Miniature Dachshund, whereas (GGCGCG) ${ }_{3}$ was extremely high in the Shih Tzu, Papillon, and Yorkshire Terrier (Table 2). Since the three NOG VNTR alleles were distributed widely among various dog breeds, these sequence-length polymorphisms arose long before the modern dog breeds were established several hundred years ago.

Because we collected DNA samples from visibly healthy dogs, each VNTR allele in the canine $N O G$ gene did not appear to be linked to fatal outcomes and severe pathological phenotypes in young dogs. Considering the important role of NOG in bone regeneration and homeostasis in adults (Canalis 2009), however, the three canine $N O G$ variations theoretically could be associated with susceptibility to bone or joint disorders in the lifetime of the domestic dog. Nevertheless, the polymorphisms are most likely silent with respect to function given that (1) the additional residues encoded by the repeats were inserted in the most flexible portion of the Noggin protein, (2) the segment is well removed from the ligand-binding interface, (3) the loop is expanded in other mammals with no apparent loss of function, and (4) the inserted residues are small and functionally inert in the context of a disordered loop (Supplementary Fig. 1). In contrast, polymorphisms resulting in insertion of tracts of polyglutamine in transcription factors can have a toxic 
gain-of-function effect in neurodegenerative disorders, triggering conformational changes that disrupt gene regulation (Riley and Orr, 2006) or culminate in formation of amyloid-like fibrils (Robertson et al. 2011). Indeed, insertion of glutamine repeats in Runx-2 protein, a transcription factor that regulates osteoblast differentiation, can have dramatic effects on canine skeletal morphogenesis (Fondon and Garner 2004). Introduction of amide-containing side-chains, with two functional groups capable of hydrogen bonding, can enhance interactions within multiprotein transcriptional complexes, leading to upregulation of target genes involved in skeletogenesis. Therefore, although polymorphisms within domestic dog breeds can have pronounced effects on development, the structural context and nature of the sidechain of the inserted residues appears to dictate whether the effect is functionally silent, accumulating over evolutionarily large time spans, or pronounced, capable of generating rapid diversity of form.

Acknowledgment This work was partly supported by a research project grant awarded by the Azabu University to K. Tanaka.

Open Access This article is distributed under the terms of the Creative Commons Attribution Noncommercial License which permits any noncommercial use, distribution, and reproduction in any medium, provided the original author(s) and source are credited.

\section{References}

Brunet LJ, McMahon JA, McMahon AP, Harland RM (1998) Noggin, cartilage morphogenesis, and joint formation in the mammalian skeleton. Science 280:1455-1457

Canalis E (2009) Growth factor control of bone mass. J Cell Biochem 108:769-777

Canalis E, Economides AN, Gazzerro E (2003) Bone morphogenetic proteins, their antagonists, and the skeleton. Endocr Rev 24:218-235

Fondon JW, Garner HR (2004) Molecular origins of rapid and continuous morphological evolution. Proc Natl Acad Sci USA 101:18058-18063

Gong Y, Krakow D, Marcelino J, Wilkin D, Chitayat D, Babul-Hirji R, Hudgins L, Cremers CW, Cremers FP, Brunner HG, Reinker K, Rimoin DL, Cohn DH, Goodman FR, Reardon W, Patton M, Francomano CA, Warman ML (1999) Heterozygous mutations in the gene encoding noggin affect human joint morphogenesis. Nature Genet 21:302-304

Groppe J, Greenwald J, Wiater E, Rodriguez-Leon J, Economides AN, Kwiatkowski W, Affolter M, Vale WW, Belmonte JC, Choe S (2002) Structural basis of BMP signalling inhibition by the cystine knot protein Noggin. Nature 420:636-642

Hirshoren N, Gross M, Banin E, Sosna J, Bargal R, Raas-Rothschild A (2008) P35S mutation in the NOG gene associated with Teunissen-Cremers syndrome and features of multiple NOG joint-fusion syndromes. Eur J Med Genet 51:351-357

Marcelino J, Sciortino CM, Romero MF, Ulatowski LM, Ballock RT, Economides AN, Eimon PM, Harland RM, Warman ML (2001) Human disease-causing NOG missense mutations: effects on noggin secretion, dimer formation, and bone morphogenetic protein binding. PNAS 98:1135311358

McMahon JA, Takada S, Zimmerman LB, Fan CM, Harland RM, McMahon AP (1998) Noggin-mediated antagonism of BMP signaling is required for growth and patterning of the neural tube and somite. Genes Dev 12:1438-1452

Riley BE, Orr HT (2006) Polyglutamine neurodegenerative diseases and regulation of transcription: assembling the puzzle. Genes Dev 20:2183-2192 
Robertson AL, Bate MA, Androulakis SG, Bottomly SP, Buckle AM (2011) PolyQ: a database describing the sequence and domain context of polyglutamine repeats in proteins. Nucleic Acids Res 39(Database issue):D272-D276

Sambrook J, Fritsch EF, Maniatis T (1989) Molecular cloning: a laboratory manual, 2nd edn. Cold Spring Harbor Laboratory Press, New York

Smith WC, Harland RM (1992) Expression cloning of noggin, a new dorsalizing factor localized to the Spemann organizer in Xenopus embryos. Cell 70:829-840

Valenzuela DM, Economides AN, Rojas E, Lamb TM, Nunez L, Jones P, Lp NY, Espinosa R 3rd, Brannan CI, Gilbert DJ, Copeland NG, Jenkins NA, Le Beau MM, Harland RM, Yancopoulos GD (1995) Identification of mammalian noggin and its expression in the adult nervous system. J Neurosci 15:6077-6084

Zimmerman LB, De Jesus-Escobar JM, Harland RM (1996) The Spemann organizer signal noggin binds and inactivates bone morphogenetic protein 4. Cell 86:599-606 